\title{
Prevalence of Dengue Virus Infection Among Febrile Outpatients Attending University of Maiduguri Teaching Hospital in Borno State, Nigeria
}

\author{
${ }^{1}$ T.M. Hamisu, ${ }^{2}$ A.D. El-Yuguda, ${ }^{3}$ M.B Abubakar, ${ }^{4}$ Y.M. Shettima,${ }^{5}$ M.M. Maina, \\ ${ }^{6}$ M.Y. Zanna, ${ }^{7}$ S.S. Baba, ${ }^{8}$ A. Andrew, ${ }^{9}$ i.C. Terhemen \\ Virus Research Laboratory, Department Of Veterinary Microbiology, Faculty Of Veterinary Medicine, \\ University Of Maiduguri, P.M.B 1069 Maiduguri, Nigeria.
}

\begin{abstract}
Dengue fever is a zoonotic arthropod-borne viral disease caused by Dengue fever virus (DENV) of the Genus Flavivirus and the FamilyFlaviviridaethat is endemic in Africa and beyond. The illness could be fatal especially among children and depleted patients.This study was designed to diagnose recentDengue virus infectionsamong febrile patients attending University of Maiduguri Teaching Hospital using ELISA kits for the detection of DENV IgM antibodies and NS1 antigens. Ninety one (91) venous blood samples were randomly collected from patients attending Maiduguri Teaching Hospital for malaria test between January and May, 2016. The samples were analysed using Enzyme Linked Immunosorbent Assays for dengue virus IgM antibodies (manufactured by Inverness Innovations Australia Pty Ltd) and for Dengue virus NSIantigens (manufactured by Bio-Rad, France). The results showed a prevalence rate of 37.4\% for DENV IgM antibodies and $9.9 \%$ for DENV NS1 antigens, with 3.3\% of the subjects testingpositive for both IgM and NS1 antigen. Females were observed to have higher IgM prevalence rate of $41 \%$ and males showed higher NS1 antigen prevalence rate of $11.1 \%$. The highest prevalence rate of $76.9 \%$ was recorded for IgM in the age bracket of 1-14 years. Samples from urban areas have the highest IgM antibodyprevalence rate of 41.4\%, however, rural dwellers have the highest NS1 antigen prevalence rate of $11.3 \%$. The presenting complaint with the highest IgM prevalence rate of 50\% was headache + fever + nausea, while headache only had the highest NS1 antigen of $22.2 \%$. The highest prevalence rate of 47.4\% for IgM and 50\% for NS1 antigen were recorded in March and May, 2016 respectively. This study therefore showed a high prevalence of IgM to Dengue fever virus indicating its circulation among febrile patients attending University of Maiduguri Teaching Hospital. Due to the probable misdiagnosis and subsequent treatment of dengue fever with other diseases such as malaria, dengue fever is shown to be prevalent in the study area. It is therefore recommended that febrile patients attending Maiduguri Teaching Hospital should be tested for dengue fever virus.
\end{abstract}

Keywords: Dengue Fever Virus,Enzyme linked Immunosorbent assay,IgM antibodies, NS1 antigen, Febrile patients,University of Maiduguri Teaching Hospital, Nigeria

\section{Introduction}

Dengue fever is a mosquito-borne viral infectioncaused by Dengue virusbelongingto genus flavivirus, in thefamily Flaviviridae. Dengue virus (DENV) containsnon-segmented, positive sense single stranded RNA genome [1]. The dengue virus (DENV) has four distinct serotypes. While infection with one DENV serotype typically provides livelong immunity against the same serotype, it does not provide lasting immunity against the other serotypes [2]. Dengue fever virus is primarily transmitted by Aedes eagypti [3]. The virus is maintained in the forests of Southeast Asia and Africa by transmission from female Aedes mosquitoes of species other than Aedes aegyptito her offspring and to lower primates [4].Clinical manifestations associated with dengue fever ranges from subclinical infection to the potentially fatal dengue shock syndrome [5]. Dengue infection is also associated with anomalies in certain haematological and biochemical parameters. Specifically, leucopenia, thrombocytopaenia, haemo-concentration and elevated serum transaminases can be present in acute dengue virus infection [6]. The dengue viruses are now arguably the most important arthropod-borne viruses from medical and public health perspective [7]. Currently, about 96 million of the estimated 390 million infections that occurred annually in the world, have some clinical manifestations [8]. Around 2.5 billion people in the world are at risk of infection as more than 100 countries are affected by dengue outbreaks [9]. The laboratory diagnosis of dengue is achieved by detecting either viral components or host antibodies mounted against the virus. Thus, for patients presenting early (day 1-7 of illness), the detection of viral non-structural protein 1 (NS1) in sera is recommended [10]. Dengue-specific antibodies of diagnostic importance are immunoglobulins $M$ and $\mathrm{G}$ (IgM and $\mathrm{IgG}$ ). In primary infections, dengue-specific IgM can be detectable as early as day 4 of illness and dengue-specific $\operatorname{IgG}$ by the fourteenth day [10;11]. In secondary infections both $\operatorname{IgG}$ and $\operatorname{IgM}$ appear as early as day 2 of illness [11]. 
Dengue is usually not among the differential diagnoses of acute febrile illness in most African countries because, among other reasons, malaria is the most prominent endemic febrile illness in Africa and does not require complex clinical and laboratory diagnostic facilities $[12 ; 13]$. Since the first isolation of DENV in Nigeria [14], there has been massive under-reporting of this infection possibly due to unavailability of sufficient diagnostic tools in the health institutions [15] and low awareness by health care providers despite reports of the virusbeen actively circulating in various parts of the country[16; 15].Many patients with fever are designated as having fever of unknown origin or malaria or typhoid and remain without a diagnosis even if they fail to respond to antimalarial or antityphoid drugs. Under these prevailing practices, there is a real potential of misdiagnosing dengue fever $[17 ; 18]$. This studywastherefore undertaken to determine the prevalence of dengue fever in the study area.

\section{Sample Collection}

\section{Materials And Methods}

Ninety one venous blood samples were collected into sterile plain vacutainer tubes from febrile outpatients attending University of Maiduguri Teaching Hospital from the month of January to May, 2015. The samples were kept at room temperature and sera were harvested by centrifugation at 2,000 revolutions per minute for 5 minutes. The harvested sera were stored in cryotubes at $-20{ }^{0} \mathrm{C}$ until tested.

\section{Laboratory Analyses}

Enzyme linked immunosorbent assay (ELISA) kits were used for the detection of dengue virusIgM antibodies and NS1 antigen according to the manufacturer's protocol.Briefly, a concentrated pool of dengue 1-4 antigens was diluted with antigen diluents. This is followed by an addition of equal volume of the horseradish peroxidase (HRP) conjugated monoclonal (MAb) antibody. The antigen-MAb complex was added to the assay plate and incubated. A substrate system, TMB chromagen, was added to the microwells after washing the plate. The reaction was stopped after adding stop solution $\left(1 \mathrm{~N} \mathrm{H}_{2} \mathrm{SO} 4\right)$. A colour development after stopping the reaction was an indication of positive test for anti-dengue $\operatorname{IgM}$ antibodies in the samples. For dengue NS1 antigen, both samples and controls were incubated simultaneously with the conjugate for 90 minutes at $37{ }^{\circ} \mathrm{C}$ in the microplate wells sensitized with MAb. Formation of MAb-NS 1-MAb/peroxidase complex is an indication of presence of NS1 antigen in the samples and is demonstrated by distribution in each well of a chromogenic solution which initiated a colour development reaction. The enzymatic reaction was stopped by adding stop solution $\left(1 \mathrm{~N} \mathrm{H}_{2} \mathrm{SO} 4\right)$ after 30 minutes of incubation. The optical density reading obtained was proportional tothe amount of NS1 antigen in the sample. The presence of NS1 antigen in an individual sample is determined by comparing the optical density reading of the sample to the optical density of the calibrator.

\section{Data Analyses}

Results were presented in tables and charts. All the samples with index value of $<0.9$ were considered negative, while samples with index values of $0.9-1.1$ and $>1.1$ were considered equivocal and positive respectively for dengue IgM ELISA test. For dengue NS1 antigen, samples with ratio value of $<0.5$ were considered negative (or nonreactive); samples with ratio value of $0.50 \leq$ ratio $<1.00$ were considered equivocal to dengue NS1 and samples with ratio value of $\geq 1.00$ were considered positive (reactive to dengue NS 1 antigen).

\section{Result}

Out of the 91 samples collected,34 (37.4\%) samples were positive for DENV IgM and 9 (9.9\%) were reactive toDENV NS1 antigen.The gender distribution of the positive samples showed the females had DENV prevalence rates of $41.1 \%$ for $\operatorname{IgM}$ and $9.6 \%$ for NS1 antigen, while the males had22.2\% $\operatorname{IgM}$ and $11.1 \%$ for NS1antigen (Table 1). Table 2 shows the distribution of DENV IgM and NS1 antigen prevalence rates based on age. The age group $1-<15$ years recorded the highest prevalence rate $(76.9 \%)$ for IgM, while the age group 15 $<30$ had the highest DENV NS1 antigen prevalence. The IgM prevalence rate for DENV among the subjects was observed to be higher among the extreme age groups of $1-<15(76.9 \%)$ and $\geq 60(59 \%)$, followed by the 15 $<30(40 \%)$ and $45-<60(31.8 \%)$, and the least was in the $30-<45(16.7 \%)$. However, the DENV NS1 antigen prevalence was highest among the age group 15-<30 (20\%), followed by the 30-<45 (8.3\%) and $1-<15(7.7 \%)$. The distribution of DENV prevalence rates among different localities showed the rural areas has $35.5 \%$ and $11.3 \%$ for IgM antibodies and NS1 antigens respectively, while urban areas recorded $41.4 \%$ and $6.8 \%$ for $\operatorname{IgM}$ antibodies and NS1 antigens respectively (Table 3). No statistical difference $(\mathrm{p}<0.05)$ noted in the prevalence of DENV among the two localities. Table 4 shows patients with different categories of complaint among which those presenting with headache + fever + nausea recorded the highest IgM prevalence rate of 50\%, while the highest NS1 antigen prevalence rate of $22.2 \%$ was recorded in patients with chief complaint of headache only. The optical density readings for the ELISA IgM antibodies and NS1 antigens showed the positive samples with 
OD values $05-<1.0$ have $58.2 \%$ and $86 \%$ frequencies for IgM and NS1 antigen respectively, by the OD values $1.0-<1.5$ (Figure 1). Whereas the NS1 antigen positive samples gave OD values of up to $\leq 1.5$, the IgM antibodies positive samples showed OD values of up to 2.5 . The prevalence rates of DENV depicted by IgM antibodies only, NS1 antigen only or combined IgM antibodies and NS1 antigens is presented in table 5. The samples showing IgM antibodies only scored 37.4\%, NS1 antigen only 9.9\% and combined IgM antibodies and NS1 antigen $3.3 \%$. The monthly distribution of the DENV prevalence rates showed the month of March recorded the highest prevalence rate for IgM antibodies of 47.6\%. For NS1 antigen, the highest prevalence of $50 \%$ was recorded in May (Table 6).

Table 1: Prevalence of dengue IgM and NS1 antigen base on sex distribution among febrile patients attending University of Maiduguri Teaching Hospital (UMTH)

\begin{tabular}{|l|l|l|l|}
\hline Sex & No. Tested & No. (\%) positive for IgM & No. (\%) positive for NS 1 \\
\hline Male & 18 & $4(22.2)$ & $2(11.1)$ \\
\hline Female & 73 & $30(41.1)$ & $7(9.6)$ \\
\hline Total & 91 & $34(37.4)$ & $9(9.9)$ \\
\hline
\end{tabular}

Table 2: Prevalence of dengue IgM and NS1 antigen based on age bracket among febrile patients attending University of Maiduguri Teaching Hospital (UMTH)

\begin{tabular}{|l|l|l|l|}
\hline Age (in years) & No. Tested & No. $(\%)$ positive for IgM & No. (\%) positive for NS1 \\
\hline $\mathbf{1 -}<15$ & 13 & $10(76.9)$ & $1(7.7)$ \\
\hline $15-<30$ & 30 & $12(40)$ & $6(20)$ \\
\hline $30-<45$ & 24 & $4(16.7)$ & $2(8.3)$ \\
\hline $45-<60$ & 22 & $7(31.8)$ & $0(0)$ \\
\hline$\geq 60$ & 2 & $1(50)$ & $0(0)$ \\
\hline Total & 91 & $34(37.4)$ & $9(9.9)$ \\
\hline
\end{tabular}

Table 3:Prevalence of dengue virus IgM and NS1 antigen based on location among febrile patients attending

\begin{tabular}{|l|l|l|l|}
\hline Location & No. Tested & No. (\%) positive for IgM & No. $(\%)$ positive for NS1 \\
\hline Rural & 62 & $22(35.5)$ & $7(11.3)$ \\
\hline Urban & 29 & $12(41.4)$ & $2(6.9)$ \\
\hline Total & 91 & $34(37.4)$ & $9(9.9)$ \\
\hline
\end{tabular}

Table 4: Prevalence of dengue virus IgM and NS1 antigen based on clinical complains presented among febrile patients attending UMTH

\begin{tabular}{|l|l|l|l|}
\hline Complains & No. Tested & No. $(\%)+$ ve for IgM & No. $(\%)+$ ve for NS1 \\
\hline Headache & 9 & $4(44.4)$ & $2(22.2)$ \\
\hline Headache + fever & 38 & $16(42.1)$ & $1(2.6)$ \\
\hline Headache + fatigue & 38 & $11(29.0)$ & $5(13.2)$ \\
\hline Headache + fever + nausea & 6 & $3(50.0)$ & $1(16.7)$ \\
\hline Total & 91 & $34(37.4)$ & $9(9.9)$ \\
\hline
\end{tabular}

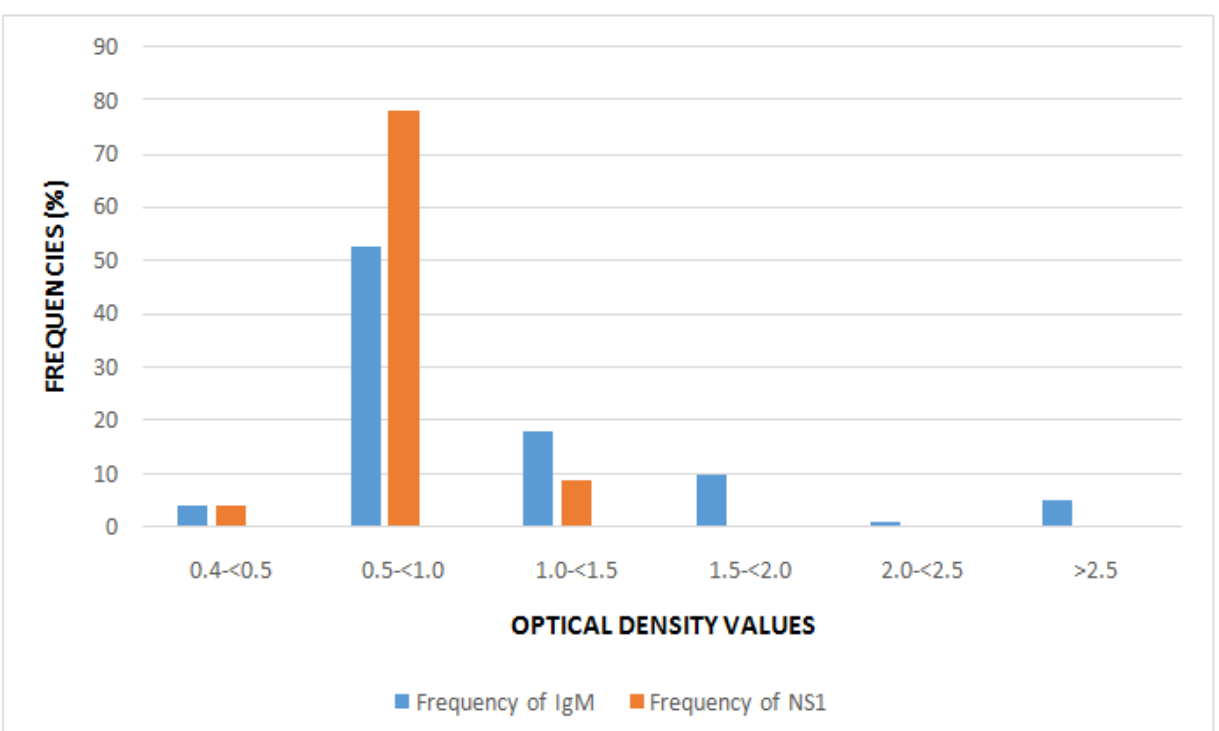

Figure 1: Histogram showing prevalence of dengue virus IgM and NS1 antigen based on recorded optical density among febrile patients attending 
Table 5: Prevalence of dengue virus monotypic and polytypic infection with IgM, NS1 and IgM + NS1 among febrile patients attending UMTH

\begin{tabular}{|l|l|l|}
\hline IgM antibodies only & NS1 antigen only & IgM + NS1 \\
\hline $34(37.4 \%)$ & $9(9.9 \%)$ & $3(3.3 \%)$ \\
\hline
\end{tabular}

Table 6: Monthly distribution of dengue virus infection among febrile patients attending UMTH

\begin{tabular}{|l|l|l|l|}
\hline Months & No. Tested & No. $(\%)$ positive for IgM & No. (\%) positive for NS1 \\
\hline January & 20 & $5(25)$ & $5(25)$ \\
\hline February & 22 & $10(45.5)$ & $0(0)$ \\
\hline March & 21 & $10(47.6)$ & $1(4.8)$ \\
\hline April & 22 & $7(31.8)$ & $0(0)$ \\
\hline May & 6 & $2(33.3)$ & $3(50)$ \\
\hline Total & 91 & $34(37.4)$ & $9(9.9)$ \\
\hline
\end{tabular}

\section{Discussion}

The high seroprevalence of DENV infection among febrile patients observed in this study goes to show the endemicity of DENV infection in the study area. This finding has further supported the previous reports of misdiagnosis of this disease among patients in the study area. The DENV IgM antibody prevalence rate of $37.4 \%$ recorded in this study is higher than the $17.2 \%$ reported by Oladipo et. al.[18] in a study conducted in Ogbomosho, Nigeria, 30.8\% reported by Faneye et al.[19] and the 0.6\% reported by Baba et al.[20] all in Nigeria. However, this result is lower than the $51.9 \%$ observed by Bello et al.[21]. In addition, this study recorded a prevalence rate of $9.9 \%$ for DENV NS1 antigen that is higher than the $2.2 \%$ reported in Jos, Nigeria [16] and 5\% in Mali [22] and also lower than the 35\% in Ibadan Nigeria[23].These differences could be due to differences in the geographical locations of the study sites and the time of the year this study was carried out. Although this study was carried out during the dry season between January and May, 2016, however lack of rainfall doesn't preclude presence of stagnant water bodies occasioned by poor drainage and waste disposal systems conditions that favour the Aedes spp. mosquito to breeds in this area.

The socio-demographic characteristics of the subjects under studyrevealed that females showed higher prevalence rate of $41 \%$ for DENV IgM as compared to the males who recorded higher prevalence rate $(11.1 \%)$ for DENV NS1 antigen.The higher IgM prevalence recorded in females is in agreement with Bello et al.[21] who reported a prevalence rate of $51.09 \%$ in females against $48.9 \%$ in males in Kaduna state, Nigeria. However, this observation contrasts that of Oladipo et al. [18] who reported a higher prevalence in males than females. The differences in prevalence rates between the genders could be attributed to differences in sample size in the different studies. The higher prevalence of dengue NS1 in males than females reported in this study is in disagreement with Dawurung et al.[16] who reported higher prevalence of dengue NS1 in females than males in Jos, Nigeria. The age bracket 1-14 years recorded the highest prevalence rate (76.9\%) for DENV IgM antibodies. This result, although higher than the 28.6\% reported by Oladipo et al.[18] and 35\% by Garg et al.[24], tallies with their observation on the age bracket that recorded the highest prevalence rates. The findings of this study also agrees with the reports of Shah et al.[25] and Anderson et al.[26]who also observed a higher prevalence of dengue virus among age group 1-15 years. The higher prevalence in this age bracket may not be unconnected with the outdoor activities of this age group. The age bracket 15-29 has the highest NS1 antigen prevalence rate $(20 \%)$ among the various age brackets sampled.

In this study, subjects from urban areas recorded the higher DENV IgM antibody prevalence rate of 41.4\%. This is in agreement with Oladipo et al.[18]in Nigeria and Collenberg et al. [27] in Burkina Faso, who documented higher DENV prevalence rates among urban dwellers. This could be related to poor urbanization planning and poor sanitary conditions associated with cities in most African countries[14]. However, the highest prevalence rate of NS1 antigen (11.3\%) observed in this study was from rural areas. This may not be unconnected with their exposure due to various farming activities in which rural dwellers are known for.

Result from this study showed that patients with multiple complaint of headache + fever + nausea recorded the highest IgM prevalence rate of 50\% and those with complaint of headache alone had the highest prevalence rate $(22.2 \%)$ of DENV NS1 antigen. Although differences between these results may likely be influenced by the differences in the number of patients tested per complaint, but it shows that many cases of dengue fever virus may be misdiagnosed for other ailments. The result of this study indicates that up to $3.3 \%$ of the subjects were suffering from DENV infection as at the time of collecting the samples. This is evidenced by the detection of DENV IgM antibodies and NS1 antigens in same subject. It was also observed in this study that the cases of DENV infection is increasing in the study area, going by previous studies that showed the infection to occur only during the rainy season [15]. The authors suggest that Public enlightenment and periodic nationwide surveillance and screening of febrile patients for dengue fever be carried out. 


\section{Acknowledgement}

The authors acknowledge with thanks the technical assistance of the WHO Polio Laboratory University of Maiduguri Teaching Hospital Nigeria.

\section{References}

[1]. Chamber TJ, Hahn CS, GallerR, Rice CM. Flavivirus genome, organization, expression and replication. Annual Review of Microbiology. 1990; 44: 649-688.

[2]. Bain SV. Dengue fever: An emerging infectious disease in The Bahamas. The International Journal of Bahamian Studies.2011; 17(2): 67-72doi:10.15362/ijbs.v17i2.155.

[3]. Weaver SC, Reisen WK. Present and future arboviral threats. Antiviral Resarch. 2010; 85: 328-345. DOI: 10.1016/j.antiviral.2009.10.008

[4]. Gubler DJ. Dengue Viruses. In: Desk Encyclopedia of Human and Medical Virology, Mahy, B.W.J., and Van Regenmortel, M.H.V (Eds.), Academic Press, Oxford, ISBN -10: 0123785596. 2010. pp: 372-82

[5]. Guzman MG, Halstead SC, Artsob H, Buchy P, Farrar J, Gubler DJ, et al. Dengue: a continuing global threat. Nature Reviews Microbiology. 2010; 8(12):S7-S16. doi:10.1038/nrmicro2460

[6]. Azin FR, Goncalves RP, Pitombeira MH, Lima DM, Branco IC. Dengue: Profile of hematological and biochemical dynamics. Revista Brasileira de Hematologia e Hemoterapia. 2012; 34:36-41. doi:10.5581/1516-8484.20120012

[7]. Rothman AL. Dengue: defining protective versus pathogenic immunity. The Journal of Clinical Investigation, 2004; 113 (7): 946951.

[8]. Bhatt S, Gething PW, Brady OJ, Messina JP, Farlow AW, Moyes CL, et al.The global distribution and burden of dengue. Nature. 2013; 496(7446):504-507

[9]. Knipe DM, Howley PM. Fields Virology. 6th ed. Philadelphia: Walters Klewer/Lippincott, Williams, and Wilkins (ed). 2014.

[10]. Simmons CP, Jeremy J, Farrar JJ, Chau NV, Wills B. Dengue. The New England Journal of medicine. 2012;366(15): 1423-1432

[11]. Wang SW, Sekaran SD. Early diagnosis of dengue infections using a commercial dengue duo rapid test kit for the detection of NS1, IgM and IgG. The American Journal of Tropical Medicine and Hygiene, 2010; 83(3): 690-695

[12]. Sang RC. Dengue in Africa. In: Report of the scientific working group meeting on dengue. Geneva, October 1-5, 2006. WHO Special Program for Research and Training in Tropical Diseases. 2007. p. 50-52

[13]. World Health Organization.Dengue: guidelines for diagnosis, treatment, prevention and control, Geneva. 2009.

[14]. Amarasinghe A, Kuritsky JN, Letson GW, Margolis HS. Dengue Virus Infection in Africa. Emerging Infectious diseases. 2011; 17(8):1349-1354

[15]. Baba MM, Talle M. The effect of climate on Dengue virus infection in Nigeria. New York Science Journal, 2011 ; 4: 28-33.

[16]. Dawurung JS, Baba MM, Stephen G, Jonas SC Bukbuk DN. Serological evidence of acute Dengue virus infection among febrile patients attending Plateau State specialist hospital Jos, Nigeria. Report and Opinion. 2010; 2: 71-76

[17]. Ayukekbong JA. Dengue Virus in Nigeria: Current Status and Future Perspective. British Journal of Virology, 2014; 1(3): 106-111

[18]. Oladipo EK, Amanetu C, Gbadero TA, Oloke JK. Detectable anti-dengue virus IgM antibodies among healthy individuals in Ogbomoso, Oyo state, Nigeria. American Journal of Infectious Diseases. 2014; 10 (2):64-67.

[19]. Faneye A, Idika N, Motayo BO, Adesanmi A, Afocha E. Serological evidence of recent dengue virus infection among febrile children in a semi-arid zone. American Journal of Infectious Disease. 2013; 9: 7-10

[20]. Baba MM, Sharon MF, Vorndam AV, Adeniji JA, Diop O. Dengue virus infections in patients suspected of malaria/thyphoid in Nigeria. Journal of American Science, 2009; 5: 129-134

[21]. Bello OA, Aminu M, Jatau ED. Seroprevalence of IgM Antibodies to Dengue Fever Virus among Patients Presenting with Symptoms of Fever in Some Hospitals in Kaduna State, Nigeria. International Journal of Science and Research, 2014; 5(3):12551259

[22]. Phoutrides EK, Coulibaly MB, George CM, Sacko A, Traore S, Bessoff K, et al. Dengue Virus Seroprevalence Among Febrile Patients in Bamako, Mali: Results of a 2006 Surveillance Study. Vector-Borne and Zoonotic Diseases.2011; 11(11): 1479-1485.

[23]. Oyero OG, Ayukekbong JA. High dengue NS1 antigenemia in febrile patients in Ibadan, Nigeria. Virus research.2014; 191: 59-61.

[24]. Garg A, Garg J, Raoy K., Upadhyay GC, Sakhuja S. Prevalence of dengue among clinically suspected febrile episodes at a teaching hospital in North India. Journal of Infectious Diseases and Immunity. 2011; 3(5):85-89.

[25]. Shah GS, Islam S, Das BK. Clinical and laboratory profile of dengue infection in children. Kathmandu University Medical Journal.2006; 13: 40-44.

[26]. Anderson KB, Chunsutiwwat S, Nisalak A, Mameen P, Libarty D. Burden of symptomatic dengue infection in children at primary school in Thailand: A prospective study. Lancet.2007; 369: 1452-59. DOI: 10.1016/S0140-6736(07)60671-0

[27]. Collenberg E, Quedraogo T, Ganamé J, Fickenscher H, Kynast-Wolf G, Becher H, et al. Seroprevalence of six different viruses among pregnant women and blood donors in rural and urban Burkina Faso: a comparative analysis.Journal of Medical Virology,78(5):683-92. 10.1002/jmv.20593 